\title{
Some Properties on Complex Functional Difference Equations
}

\author{
Zhi-Bo Huang ${ }^{1,2}$ and Ran-Ran Zhang ${ }^{3}$ \\ ${ }^{1}$ School of Mathematical Sciences, South China Normal University, Guangzhou 510631, China \\ ${ }^{2}$ Department of Physics and Mathematics, University of Eastern Finland, P.O. Box 111, 80101 Joensuu, Finland \\ ${ }^{3}$ Department of Mathematics, Guangdong University of Education, Guangzhou 510303, China
}

Correspondence should be addressed to Zhi-Bo Huang; huangzhibo@scnu.edu.cn

Received 15 January 2014; Accepted 13 March 2014; Published 24 April 2014

Academic Editor: Zong-Xuan Chen

Copyright (C) 2014 Z.-B. Huang and R.-R. Zhang. This is an open access article distributed under the Creative Commons Attribution License, which permits unrestricted use, distribution, and reproduction in any medium, provided the original work is properly cited.

\begin{abstract}
We obtain some results on the transcendental meromorphic solutions of complex functional difference equations of the form $\sum_{\lambda \in I} \alpha_{\lambda}(z)\left(\prod_{j=0}^{n} f\left(z+c_{j}\right)^{\lambda_{j}}\right)=R(z, f \circ p)=\left(\left(a_{0}(z)+a_{1}(z)(f \circ p)+\cdots+a_{s}(z)(f \circ p)^{s}\right) /\left(b_{0}(z)+b_{1}(z)(f \circ p)+\cdots+b_{t}(z)(f \circ p)^{t}\right)\right)$, where $I$ is a finite set of multi-indexes $\lambda=\left(\lambda_{0}, \lambda_{1}, \ldots, \lambda_{n}\right), c_{0}=0, c_{j} \in \mathbb{C} \backslash\{0\}(j=1,2, \ldots, n)$ are distinct complex constants, $p(z)$ is a polynomial, and $\alpha_{\lambda}(z)(\lambda \in I), a_{i}(z)(i=0,1, \ldots, s)$, and $b_{j}(z)(j=0,1, \ldots, t)$ are small meromorphic functions relative to $f(z)$. We further investigate the above functional difference equation which has special type if its solution has Borel exceptional zero and pole.
\end{abstract}

\section{Introduction and Main Results}

In this paper, a meromorphic function means meromorphic in the whole complex plane $\mathbb{C}$. For a meromorphic function $y(z)$, let $\sigma(y)$ be the order of growth and $\mu(y)$ the lower order of $y(z)$. Further, let $\lambda(y)$ (resp., $\lambda(1 / y)$ ) be the exponent of convergence of the zeros (resp., poles) of $y(z)$. We also assume that the reader is familiar with the fundamental results and the standard notations of Nevanlinna theory of meromorphic functions (see, e.g., [1]). Given a meromorphic function $y(z)$, we call a meromorphic function $a(z)$ a small function relative to $y(z)$ if $T(r, a(z))=S(r, y)=o(T(r, y))$ as $r \rightarrow \infty$, possibly outside of an exceptional set of finite logarithmic measure. Moreover, if $R(z, y)$ is rational in $y(z)$ with small functions relative to $y(z)$ as its coefficients, we use the notation $d=\operatorname{deg}_{y} R(z, y)$ for the degree of $R(z, y)$ with respect to $y(z)$. In what follows, we always assume that $R(z, y)$ is irreducible in $y(z)$.

Meromorphic solutions of complex difference equations have recently gained increasing interest, due to the problem of integrability of difference equations. This is related to the activity concerning Painlevé differential equations and their discrete counterparts in the last decades. Ablowitz et al. [2] considered discrete equations to be delay equations in the complex plane. This allowed them to analyze these equations with the methods from complex analysis. In regard to related papers concerning a more general class of complex difference equations, we may refer to [3-5]. These papers mainly dealt with equations of the form

$$
\sum_{\{J\}} \alpha_{J}(z)\left(\prod_{j \in J} f\left(z+c_{j}\right)\right)=R(z, f),
$$

where $\{J\}$ is a collection of all nonempty subsets of $\{1,2, \ldots, n\}, c_{j}(j \in J)$ are distinct complex constants, $f(z)$ is a transcendental meromorphic function, $\alpha_{J}(z)(J \in\{J\})$ are small functions relative to $f(z)$, and $R(z, f)$ is a rational function in $f(z)$ with small meromorphic coefficients. Moreover, if the right-hand side of (1) is essentially like the composite function $e \circ f$ of $f(z)$ and a rational function $e(z)$, Laine et al. reversed the order of composition; that is, they considered the composite function $f \circ e$ of $f(z)$ and a rational function $e(z)$, which resulted in a complex functional difference equation. The following theorem [5, Theorem 2.8] gives an example.

Theorem A (see [5, Theorem 2.8]). Suppose that $f(z)$ is a transcendental meromorphic solution of equation

$$
\sum_{\{J\}} \alpha_{J}(z)\left(\prod_{j \in J} f\left(z+c_{j}\right)\right)=f(p(z)),
$$


where $p(z)$ is a polynomial of degree $k \geq 2$. Moreover, one assumes that the coefficients $\alpha_{J}(z)$ are small functions relative to $f(z)$ and that $n \geq k$. Then

$$
T(r, f)=O\left((\log r)^{\alpha+\varepsilon}\right),
$$

where $\alpha=(\log n) /(\log k)$.

At this point, we briefly introduce some notations used in this paper. A difference monomial of a meromorphic function $f(z)$ is defined as

$$
f(z)^{\lambda_{0}} f\left(z+c_{1}\right)^{\lambda_{1}} \cdots f\left(z+c_{n}\right)^{\lambda_{s}}:=\prod_{j=0}^{s} f\left(z+c_{j}\right)^{\lambda_{j}},
$$

where $c_{0}=0, c_{j} \in \mathbb{C} \backslash\{0\}(j=1,2, \ldots, s)$ are distinct constants, and $\lambda_{j}(j=0,1, \ldots, s)$ are natural numbers. A difference polynomial $H(z, f(z))$ of a meromorphic function $f(z)$, a finite sum of difference monomials, is defined as

$$
H(z, f(z))=\sum_{\lambda \in I} \alpha_{\lambda}(z)\left(\prod_{j=0}^{n} f\left(z+c_{j}\right)^{\lambda_{j}}\right),
$$

where $I$ is a finite set of multi-indexes $\lambda=\left(\lambda_{0}, \lambda_{1}, \ldots, \lambda_{n}\right)$, $\alpha_{\lambda}(z)(\lambda \in I)$ are small functions relative to $f(z)$. The degree and the weight of the difference polynomial (5), respectively, are defined as

$$
\operatorname{deg}_{f}(H)=\max _{\lambda \in I}\left\{\sum_{j=0}^{n} \lambda_{j}\right\}, \quad \kappa_{f}(H)=\max _{\lambda \in I}\left\{\sum_{j=1}^{n} \lambda_{j}\right\} .
$$

Consequently, $\kappa_{f}(H) \leq \operatorname{deg}_{f}(H)$. For instance, the degree and the weight of the difference polynomial $f^{2}(z) f(z-1) f(z+1)+$ $f(z) f(z+1) f(z+2)+f^{2}(z-1) f(z+2)$, respectively, are four and three. Moreover, a difference polynomial (5) is said to be homogeneous with respect to $f(z)$ if the degree $\sum_{j=0}^{n} \lambda_{j}$ of each monomial in the sum of (5) is nonzero and the same for all $\lambda \in I$.

In the following, we proceed to prove generalizations of Theorem A and investigate some new results for the first time. We permit more general expressions on both sides of (1).

Theorem 1. Let $f(z)$ be a transcendental meromorphic solution of equation

$$
\begin{aligned}
H(z, f(z)) & =R(z, f \circ p) \\
& =\frac{a_{0}(z)+a_{1}(z)(f \circ p)+\cdots+a_{s}(z)(f \circ p)^{s}}{b_{0}(z)+b_{1}(z)(f \circ p)+\cdots+b_{t}(z)(f \circ p)^{t}}
\end{aligned}
$$

where $H(z, f(z))$ is defined as (5), $p(z)=d_{k} z^{k}+\cdots+d_{1} z+d_{0}$ is a polynomial with constant coefficients $d_{k}(\neq 0), \ldots, d_{1}, d_{0}$ and of the degree $k \geq 2$, and $a_{i}(z)(i=0,1, \ldots, s)$ and $b_{j}(z)(j=0,1, \ldots, t)$ are small meromorphic functions relative to $f(z)$ such that $a_{s}(z) b_{t}(z) \neq \equiv$. Set $d=\max \{s, t\}$. If $k d \leq$ $(n+1) \operatorname{deg}_{f}(H)$, then

$$
T(r, f)=O\left((\log r)^{\alpha+\varepsilon}\right),
$$

where $\alpha=\left(\log (n+1)+\log \operatorname{deg}_{f}(H)-\log d\right) /(\log k)$.
Similar to the proof of Theorem 1, we easily obtain the following result, which is a generation of Theorem A.

Theorem 2. Let $c_{i} \in \mathbb{C}(i=1,2, \ldots, n)$ be distinct constants and $f(z)$ be a transcendental meromorphic solution of equation

$$
\begin{aligned}
& \sum_{\{J\}} \alpha_{J}(z)\left(\prod_{j \in J} f\left(z+c_{j}\right)\right) \\
& =R(z, f \circ p) \\
& =\frac{a_{0}(z)+a_{1}(z)(f \circ p)+\cdots+a_{s}(z)(f \circ p)^{s}}{b_{0}(z)+b_{1}(z)(f \circ p)+\cdots+b_{t}(z)(f \circ p)^{t}},
\end{aligned}
$$

where $p(z)=d_{k} z^{k}+\cdots+d_{1} z+d_{0}$ is a polynomial with constant coefficients $d_{k}(\neq 0), \ldots, d_{1}, d_{0}$ and of the degree $k \geq 2$ and $a_{i}(z)(i=0,1, \ldots, s)$ and $b_{j}(z)(j=0,1, \ldots, t)$ are small functions relative to $f(z)$ such that $a_{s}(z) b_{t}(z)$ \# 0 . If $k d=$ $k \max \{s, t\} \leq n$, then

$$
T(r, f)=O\left((\log r)^{\alpha+\varepsilon}\right),
$$

where $\alpha=(\log n-\log d) /(\log k)$.

We then proceed to consider the distribution of zeros and poles of meromorphic solutions of (7). The following result indicates that solutions having Borel exceptional zeros and poles appear only in special situations.

Theorem 3. Let $c_{0}=0$, let $c_{i} \in \mathbb{C} \backslash\{0\}(i=1,2, \ldots, n)$ be distinct constants, and let $f(z)$ be a finite order transcendental meromorphic solution of equation

$$
\begin{aligned}
& \prod_{j=0}^{n} f\left(z+c_{j}\right)^{\lambda_{j}} \\
& \quad=R(z, f \circ p) \\
& \quad=\frac{a_{0}(z)+a_{1}(z)(f \circ p)+\cdots+a_{s}(z)(f \circ p)^{s}}{b_{0}(z)+b_{1}(z)(f \circ p)+\cdots+b_{t}(z)(f \circ p)^{t}},
\end{aligned}
$$

where $p(z)=d_{k} z^{k}+\cdots+d_{1} z+d_{0}$ is a polynomial with constant coefficients $d_{k}(\neq 0), \ldots, d_{1}, d_{0}$ and of the degree $k \geq 1$ and $a_{i}(z)(i=0,1, \ldots, s)$ and $b_{j}(z)(j=0,1, \ldots, t)$ are small meromorphic functions relative to $f(z)$ such that $a_{s}(z) b_{t}(z) \not \equiv$ 0. If

$$
\max \left\{\lambda(f), \lambda\left(\frac{1}{f}\right)\right\}<\sigma(f)
$$

then (11) is either of the form

$$
\prod_{j=0}^{n} f\left(z+c_{j}\right)^{\lambda_{j}}=\alpha \frac{a_{s}(z)}{b_{0}(z)}(f \circ p)^{s}
$$

or

$$
\prod_{j=0}^{n} f\left(z+c_{j}\right)^{\lambda_{j}}=\alpha \frac{a_{0}(z)}{b_{t}(z)} \frac{1}{(f \circ p)^{t}}
$$

where $\alpha \in \mathbb{C} \backslash\{0\}$ is some constant. 
Example 4. $f(z)=\cos z$ solves difference equation

$$
4 f(z)^{2} f(z+\pi)^{2}=f(2 z)^{2}+2 f(2 z)+1 .
$$

Here $p(z)=2 z$. Clearly, $\lambda(1 / f)=0<1=\lambda(f)=\sigma(f)$. This example shows that condition (12) is necessary and cannot be replaced by

$$
\min \left\{\lambda(f), \lambda\left(\frac{1}{f}\right)\right\}<\sigma(f) .
$$

Moreover, we obtain a result parallel to Theorem 5.4 in [6] for the difference case.

\section{Theorem 5. Suppose that the equation}

$$
\prod_{j=0}^{n} f\left(z+c_{j}\right)^{\lambda_{j}}=\frac{c(z)}{f(z)^{m}}, \quad m \in \mathbb{N},
$$

has a meromorphic solution of finite order, where $c_{0}=0, c_{j} \in$ $\mathbb{C} \backslash\{0\}(j=1,2, \ldots, n)$ are distinct constants, and $c(z)$ is a nontrivial meromorphic function. If $f(z)$ has only finitely many poles, then $f(z)=D(z) e^{E(z)}$, where $D(z)$ is a rational function and $E(z)$ is a polynomial, if and only if $c(z)=G(z) e^{M(z)}$, where $G(z)$ is a rational function and $M(z)$ is a polynomial.

Example 6. Difference equation

$$
f(z)^{2} f(z+1) f(z-1)=\left(\frac{1}{z^{4}\left(z^{2}-1\right)} e^{6 z}\right) \cdot \frac{1}{f(z)^{2}}
$$

of the type (17) is solved by $f(z)=e^{z} / z$. Here, $f(z)=e^{z} / z$ and $c(z)=e^{6 z} / z^{4}\left(z^{2}-1\right)$ satisfy Theorem 5 .

As an application of Theorem 3, we obtain the following.

Theorem 7. Let $c \in \mathbb{C} \backslash\{0\}$ and let $f(z)$ be a finite order transcendental meromorphic solution of equation

$$
\begin{aligned}
f(z+c) & =R(z, f \circ p) \\
& =\frac{a_{0}(z)+a_{1}(z)(f \circ p)+\cdots+a_{s}(z)(f \circ p)^{s}}{b_{0}(z)+b_{1}(z)(f \circ p)+\cdots+b_{t}(z)(f \circ p)^{t}},
\end{aligned}
$$

where $p(z)=d_{k} z^{k}+\cdots+d_{1} z+d_{0}$ is a polynomial with constant coefficients $d_{k}(\neq 0), \ldots, d_{1}, d_{0}$ and of the degree $k \geq 2$ and $a_{i}(z)(i=0,1, \ldots, s)$ and $b_{j}(z)(j=0,1, \ldots, t)$ are small meromorphic functions relative to $f(z)$ such that $a_{s}(z) b_{t}(z)$ \# 0 . Then $f(z)$ has at most one Borel exceptional value.

If the degree $k$ of polynomial $p(z)$ is 1 in Theorem 7 , the result does not hold. For example, we have the following.

Example 8. $f(z)=\tan z$ solves difference equation

$$
f(z+1)=\frac{f(z)+\tan 1}{1-(\tan 1) f(z)}
$$

of the type (19). Obviously, $f(z)$ has two Borel exceptional values $\pm i$.

If we remove the assumption $\max \{\lambda(f), \lambda(1 / f)\}<\sigma(f)$ used in Theorem 3, we obtain a result similar to Theorem 12 in [4].
Theorem 9. Let $f(z)$ be a transcendental meromorphic solution of equation

$$
\begin{aligned}
H(z, f(z)) & =R(z, f) \\
& =\frac{a_{0}(z)+a_{1}(z) f(z)+\cdots+a_{s}(z) f(z)^{s}}{b_{0}(z)+b_{1}(z) f(z)+\cdots+b_{t}(z) f(z)^{t}},
\end{aligned}
$$

where $H(z, f(z))$ is defined as (5) and $a_{i}(z)(i=0,1, \ldots, s)$ and $b_{j}(z)(j=0,1, \ldots, t)$ are small meromorphic functions relative to $f(z)$ such that $a_{s}(z) b_{t}(z) \not \equiv 0$. If $d:=\max \{s, t\}>$ $(n+1) \operatorname{deg}_{f}(H)$, then $\sigma(f)=\infty$.

In fact, the following examples show that the assertion of Theorem 9 does not remain valid identically if $d \leq(n+$ 1) $\operatorname{deg}_{f}(H)$.

Example 10. $f(z)=\exp \left\{e^{z}\right\} / z$ solves the difference equation

$$
\begin{gathered}
(z-\pi i)(z+\log 2-\pi i) f(z) f(z-\pi i) f(z+\log 2-\pi i) \\
+(z+\log 8) f(z+\log 8)=\frac{1+z^{11} f(z)^{10}}{z^{3} f(z)^{2}}
\end{gathered}
$$

Clearly, $d=10<(3+1) \cdot 3=(n+1) \operatorname{deg}_{f}(H)$ and $\sigma(f)=\infty$. Example 11. $f(z)=\tan z$ satisfies the difference equation

$$
f\left(z+\frac{\pi}{4}\right)+f\left(z-\frac{\pi}{4}\right)=\frac{4 f(z)}{1-f(z)^{2}} .
$$

Obviously, $d=2<(2+1) \times 1=(n+1) \operatorname{deg}_{f}(H)$ and $\sigma(f)=1$.

Example 12 (see [7, pages 103-106] and [8, page 8]). The following difference equation,

$$
f(z+1)=\alpha f(z)(1-f(z)), \quad \alpha \neq 0,
$$

derives from a well-known discrete logistic model in biology. It has been proved that all other meromorphic solutions are of infinite order, apart from the constant solutions $f(z) \equiv 0$ and $f(z)=(\alpha-1) / \alpha$. For instance, (24) has one-parameter families of entire solutions of infinite order:

$$
\begin{gathered}
f(z)=\frac{1}{2}\left(1-\exp \left(A e^{z \log 2}\right)\right), \quad A \in \mathbb{C} \backslash\{0\}, \alpha=2, \\
f(z)=\sin ^{2}\left(B e^{z \log 2}\right), \quad B \in \mathbb{C} \backslash\{0\}, \alpha=4 .
\end{gathered}
$$

Here, $d=2=(1+1) \times 1=(n+1) \operatorname{deg}_{f}(H)$.

Example 13. $f(z)=z$ solves the difference equation

$$
f(z+1)=\frac{1-f(z)^{2}}{-z^{2}-z+1+f(z)^{2}} .
$$

We get $d=2=(1+1) \times 1=(n+1) \operatorname{deg}_{f}(H)$ and $\sigma(f)=0$.

If the difference polynomial in the left-hand side of (21) is homogeneous, we further obtain the following theorem. 
Theorem 14. Let $f(z)$ be a transcendental meromorphic solution of (21), where $H(z, f(z))$ is defined as (5) and $a_{i}(z)(i=$ $0,1, \ldots, s)$ and $b_{j}(z)(j=0,1, \ldots, t)$ are small meromorphic functions relative to $f(z)$ such that $a_{s}(z) b_{t}(z)$ \# 0 . Suppose that $H(z, f)$ is homogeneous and has at least one difference monomial of type

$$
\prod_{j=0}^{n} f\left(z+c_{j}\right)^{\lambda_{j}}, \quad\left(\lambda_{j} \in \mathbb{N}_{+}, j=0,1, \ldots, n\right) .
$$

If $d:=\max \{s, t\}>3 \operatorname{deg}_{f}(H)$, then $\sigma(f)=\infty$.

\section{Proof of Theorem 1}

We need some preliminaries to prove Theorem 1 .

Lemma 15 (see [9, Lemma 4]). Let $f(z)$ be a transcendental meromorphic function and let $p(z)=d_{k} z^{k}+\cdots+d_{1} z+$ $d_{0}\left(d_{k} \neq 0\right)$ be a polynomial of degree $k$. Given $0<\delta<\left|d_{k}\right|$, denote $\nu:=\left|d_{k}\right|+\delta$ and $\mu:=\left|d_{k}\right|-\delta$. Then, given $\varepsilon>0$ and $a \in \mathbb{C} \cup\{\infty\}$, one has, for all $r \geq r_{0}>0$,

$$
\begin{gathered}
k n\left(\mu r^{k}, a, f\right) \leq n(r, a, f \circ p) \leq k n\left(\nu r^{k}, a, f\right), \\
\begin{aligned}
& N\left(\mu r^{k}, a, f\right)+O(\log r) \leq N(r, a, f \circ p) \leq N\left(\nu r^{k}, a, f\right) \\
&+O(\log r), \\
&(1-\varepsilon) T\left(\mu r^{k}, f\right) \leq T(r, f \circ p) \leq(1+\varepsilon) T\left(\nu r^{k}, f\right) .
\end{aligned}
\end{gathered}
$$

Lemma 16 (see [10, Theorem B.16]). Given distinct meromorphic functions $f_{1}, \ldots, f_{n}$, let $\{J\}$ denote the collection of all nonempty subsets of $\{1,2, \ldots, n\}$, and suppose that $\alpha_{J} \in \mathbb{C}$ for each $J \in\{J\}$. Then

$$
T\left(r, \sum_{\{J\}} \alpha_{J}\left(\prod_{j \in J} f_{j}\right)\right) \leq \sum_{k=1}^{n} T\left(r, f_{k}\right)+O(1) .
$$

By denoting $f_{i+1}=f\left(z+c_{i}\right)^{\lambda_{i}}(i=0,1, \ldots, n)$ below, it is an easy exercise to prove the following result from Lemma 16.

Lemma 17. Let $f(z)$ be a meromorphic function, let $I$ be a finite set of multi-indexes $\lambda=\left(\lambda_{0}, \lambda_{1}, \ldots, \lambda_{n}\right)$, and let $\alpha_{\lambda}(z)$ be small functions relative to $f(z)$ for all $\lambda \in I$. Then the characteristic function of the difference polynomial (5) satisfies

$$
\begin{aligned}
& T\left(r, \sum_{\lambda \in I} \alpha_{\lambda}(z)\left(\prod_{j=0}^{n} f\left(z+c_{j}\right)^{\lambda_{j}}\right)\right) \\
& \quad \leq(n+1) \operatorname{deg}_{f}(H) T(r+C, f)+S(r, f),
\end{aligned}
$$

where $C=\max \left\{\left|c_{1}\right|,\left|c_{2}\right|, \ldots,\left|c_{n}\right|\right\}$.

Lemma 18 (see [11, Lemma 5]). Let $g(r)$ and $h(r)$ be monotone nondecreasing functions on $[0, \infty)$ such that $g(r) \leq h(r)$ for all $r \notin E \cup[0,1]$, where $E \subset(1, \infty)$ is a set of finite logarithmic measure. Let $\alpha>1$ be a given constant. Then there exists an $r_{0}=r_{0}(\alpha)>0$ such that $g(r) \leq h(\alpha r)$ for all $r \geq r_{0}$.
Lemma 19 (see [12, Lemma 3]). Let $\psi(r)$ be a function of $r\left(r \geq r_{0}\right)$, positive and bounded in every finite interval.

(i) Suppose that $\psi\left(\mu r^{m}\right) \leq A \psi(r)+B\left(r \geq r_{0}\right)$, where $\mu(\mu>0), m(m>1), A(A \geq 1)$, and $B$ are constants. Then $\psi(r)=O\left((\log r)^{\alpha}\right)$ with $\alpha=(\log A) /(\log m)$, unless $A=1$ and $B>0$; and if $A=1$ and $B>0$, then for any $\varepsilon>0, \psi(r)=O\left((\log r)^{\varepsilon}\right)$.

(ii) Suppose that (with the notation of (i)) $\psi\left(\mu r^{m}\right) \geq$ $A \psi(r)\left(r \geq r_{0}\right)$. Then for all sufficiently large values of $r, \psi(r) \geq K(\log r)^{\alpha}$ with $\alpha=(\log A) /(\log m)$ for some positive constant $K$.

Proof of Theorem 1. For any $\varepsilon(0<\varepsilon<1)$, we may apply Valiron-Mohon'ko lemma, Lemmas 15 and 17, and (5) and (7) to conclude that

$$
\begin{aligned}
& d(1-\varepsilon) T\left(\mu r^{k}, f\right) \\
& \leq d T(r, f \circ p)+S(r, f) \\
&= T\left(r, \frac{a_{0}(z)+a_{1}(z)(f \circ p)+\cdots+a_{s}(z)(f \circ p)^{s}}{b_{0}(z)+b_{1}(z)(f \circ p)+\cdots+b_{t}(z)(f \circ p)^{t}}\right) \\
&+S(r, f) \\
&=T\left(r, \sum_{\lambda \in I} \alpha_{\lambda}(z)\left(\prod_{j=0}^{n} f\left(z+c_{j}\right)^{\lambda_{j}}\right)\right)+S(r, f) \\
& \leq(n+1) \operatorname{deg}_{f}(H) T(r+C, f)+S(r, f) \\
& \leq(n+1) \operatorname{deg}_{f}(H)(1+\varepsilon) T(r+C, f)
\end{aligned}
$$

holds for all sufficiently large $r$, possibly outside of an exceptional set of finite logarithmic measure, where $C=$ $\max \left\{\left|c_{1}\right|,\left|c_{2}\right|, \ldots,\left|c_{n}\right|\right\}$ and $\mu$ is defined as Lemma 15. Now, we may apply Lemma 18 to deal with the exceptional set and conclude that, for every $\eta>1$, there exists an $r_{0}>0$ such that

$$
d(1-\varepsilon) T\left(\mu r^{k}, f\right) \leq(n+1) \operatorname{deg}_{f}(H)(1+\varepsilon) T(\eta r, f)
$$

holds for all $r \geq r_{0}$. Denote $\omega=\eta r$. Then (32) can be written in the form

$$
T\left(\frac{\mu}{\eta^{k}} \omega^{k}, f\right) \leq \frac{(n+1) \operatorname{deg}_{f}(H)(1+\varepsilon)}{d(1-\varepsilon)} T(\omega, f) .
$$

Since $d k \leq(n+1) \operatorname{deg}_{f}(H)$, we get $\left((n+1) \operatorname{deg}_{f}(H)(1+\right.$ $\varepsilon)) /(d(1-\varepsilon))>1$ for all $0<\varepsilon<1$. Thus, we now apply Lemma 19(i) to conclude that

$$
\begin{gathered}
T(r, f)=O\left((\log r)^{\alpha+\varepsilon}\right), \\
\alpha=\frac{\log \left((n+1) \operatorname{deg}_{f}(H)(1+\varepsilon) / d(1-\varepsilon)\right)}{\log k} \\
=\frac{\log (n+1)+\log \operatorname{deg}_{f}(H)-\log d}{\log k}+o(1) .
\end{gathered}
$$

The proof of Theorem 1 is completed. 


\section{Proof of Theorems 3 and 5}

We again need some preliminaries.

Lemma 20 (see [13, Theorem 1.5]). Suppose that $f_{j}(z)(j=$ $1,2, \ldots, n)(n \geq 2)$ are meromorphic functions and $g_{j}(z)(j=$ $1,2, \ldots, n)$ are entire functions satisfying the following conditions.

(1) $\sum_{j=1}^{n} f_{j}(z) e^{g_{j}(z)}=0$.

(2) $g_{j}(z)-g_{k}(z)$ are not constants for $1 \leq j<k \leq n$.

(3) For $1 \leq j \leq n, 1 \leq h<k \leq n$,

$$
T\left(r, f_{j}\right)=o\left\{T\left(r, e^{g_{h}-g_{k}}\right)\right\} \quad(r \longrightarrow+\infty, r \notin E),
$$

where $E \subset(1,+\infty)$ is of finite linear measure or finite logarithmic measure.

Then $f_{j}(z) \equiv 0(j=1,2, \ldots, n)$.

Lemma 21 (see $\left[14\right.$, Theorem 4]). Let $F(z), P_{n}(z), \ldots, P_{0}(z)$ be polynomials such that $F P_{n} P_{0} \not \equiv 0$ and then every finite order transcendental meromorphic solution $f(z)$ of equation

$$
P_{n}(z) f(z+n)+\cdots+P_{1}(z) f(z+1)+P_{0}(z) f(z)=F(z)
$$

satisfies $\lambda(f)=\sigma(f) \geq 1$.

Remark 22. Replacing $j$ by $c_{j}(j=1,2, \ldots, n)$, where $c_{j}(j=$ $1,2, \ldots, n)$ are distinct nonzero complex constants, Lemma 21 remains valid.

Proof of Theorem 3. Let $\tau$ be the multiplicity of pole of $f(z)$ at the origin, and let $q(z)$ be a canonical product formed with nonzero poles of $f(z)$. Since $\max \{\lambda(f), \lambda(1 / f)\}<\sigma(f)$, then $h(z)=z^{\tau} q(z)$ is an entire function such that

$$
\sigma(h)=\lambda\left(\frac{1}{f}\right)<\sigma(f)<+\infty
$$

and $g(z)=h(z) f(z)$ is a transcendental entire function with

$$
\begin{gathered}
T(r, g)=T(r, f)+S(r, f), \quad \sigma(g)=\sigma(f), \\
\lambda(g)=\lambda(f) .
\end{gathered}
$$

If $q(z)$ is a polynomial, we obtain quickly that $\sigma(h \circ p)=$ $0<\sigma(g \circ p)$. Otherwise, we conclude from the last assertion of Lemma 15, (37), and (38) that

$$
\sigma(h \circ p)=k \sigma(h)=k \lambda\left(\frac{1}{f}\right)<k \sigma(g)=\sigma(g \circ p) \text {. }
$$

Therefore,

$$
T(r, h \circ p)=S(r, g \circ p)
$$

Now, substituting $f(z)=g(z) / h(z)$ into (11), we conclude that

$$
\begin{aligned}
& \frac{(h \circ p)^{s-t}}{\prod_{j=0}^{n} h\left(z+c_{j}\right)^{\lambda_{j}}}\left(\prod_{j=0}^{n} g\left(z+c_{j}\right)^{\lambda_{j}}\right) \\
& =\left(a_{0}(z)(h \circ p)^{s}+a_{1}(z)(h \circ p)^{s-1}(g \circ p)\right. \\
& \left.\quad+\cdots+a_{s}(z)(g \circ p)^{s}\right) \\
& \times\left(b_{0}(z)(h \circ p)^{t}+b_{1}(z)(h \circ p)^{t-1}(g \circ p)\right. \\
& \left.\quad+\cdots+b_{t}(z)(g \circ p)^{t}\right)^{-1} .
\end{aligned}
$$

Obviously, it follows from (37)-(40) and Lemma 15 that

$$
\begin{aligned}
T\left(r, \frac{1}{\prod_{j=0}^{n} h\left(z+c_{j}\right)^{\lambda_{j}}}\right) & =S(r, g \circ p), \\
T\left(r,(h \circ p)^{s-t}\right) & =S(r, g \circ p), \\
T\left(r, a_{u}(z)(h \circ p)^{s-u}\right) & =S(r, g \circ p), \quad u=0,1, \ldots, s, \\
T\left(r, b_{v}(z)(h \circ p)^{t-v}\right) & =S(r, g \circ p), \quad v=0,1, \ldots, t .
\end{aligned}
$$

Denoting $A(z)=(h \circ p)^{s-t} / \prod_{j=0}^{n} h\left(z+c_{j}\right)^{\lambda_{j}}$, we get from (42) that

$$
T(r, A)=S(r, g \circ p) .
$$

Since zeros and poles are Borel exceptional values of $f(z)$ by (12), we may apply a result due to Whittaker; see [15, Satz 13.4 , to deduce that $f(z)$ is of regular growth. Thus, we use Lemma 15 and (12) again to get

$$
T\left(r, \frac{f^{\prime}}{f}\right)=\bar{N}(r, f)+\bar{N}\left(r, \frac{1}{f}\right)+S(r, f)=S(r, g \circ p) \text {. }
$$

Similarly, if we set $B(z)=A(z)\left(\prod_{j=0}^{n} g\left(z+c_{j}\right)^{\lambda_{j}}\right)$, we also deduce from the lemma of the logarithmic derivative, Lemma 15, (12), (38), and (43) that

$$
T\left(r, \frac{B^{\prime}}{B}\right)=T\left(r, \frac{A^{\prime}}{A}+\sum_{j=0}^{n} \lambda_{j} \frac{g^{\prime}\left(z+c_{j}\right)}{g\left(z+c_{j}\right)}\right)=S(r, g \circ p) \text {. }
$$

Denoting $F(z)=g \circ p$,

$$
\begin{aligned}
P(z, F)= & \frac{a_{0}(z)}{a_{s}(z)}(h \circ p)^{s} \\
& +\frac{a_{1}(z)}{a_{s}(z)}(h \circ p)^{s-1} F(z)+\cdots+F(z)^{s}, \\
Q(z, F)= & \frac{b_{0}(z)}{b_{t}(z)}(h \circ p)^{t} \\
& +\frac{b_{1}(z)}{b_{t}(z)}(h \circ p)^{t-1} F(z)+\cdots+F(z)^{t} .
\end{aligned}
$$


Therefore, we deduce from Lemma 15 and (42) that the coefficients of $P(z, F)$ and $Q(z, F)$ are small functions relative to $F(z)$. Thus, (41) can be written in the form

$$
\frac{b_{t}(z)}{a_{s}(z)} B(z)=\frac{P(z, F)}{Q(z, F)}:=u(z, F) .
$$

Denoting

$$
\psi(z)=\frac{F^{\prime}(z)}{F(z)}, \quad U(z)=\frac{u^{\prime}(z, F)}{u(z, F)},
$$

we get $T(r, U)=S(r, g \circ p)$ from (45) and (47). We also conclude from the lemma of logarithmic derivative, Lemma 15, and (12) that

$$
\begin{aligned}
T(r, \psi) & =T\left(r, \frac{F^{\prime}}{F}\right)=m\left(r, \frac{F^{\prime}}{F}\right)+N\left(r, \frac{F^{\prime}}{F}\right) \\
& \leq \bar{N}(r, F)+\bar{N}\left(r, \frac{1}{F}\right)+S(r, F) \\
& =\bar{N}(r, g \circ p)+\bar{N}\left(r, \frac{1}{g \circ p}\right)+S(r, g \circ p) \\
& \leq N\left(r, \frac{1}{g \circ p}\right)+S(r, g \circ p) \\
& \leq N\left(v r^{k}, \frac{1}{g}\right)+S(r, g \circ p)=S(r, g \circ p),
\end{aligned}
$$

where $v$ is defined as Lemma 15.

Since

$$
\frac{P^{\prime} Q-P Q^{\prime}}{Q^{2}}=u^{\prime}=U u=\frac{U P}{Q},
$$

we conclude that

$$
P^{\prime} Q-P Q^{\prime}=U P Q
$$

Now, writing $F^{\prime}=\psi F$ in (51), regarding then (51) as an algebraic equation in $F$ with coefficients of growth $S(r, F)$, and comparing the leading coefficients, we deduce that

$$
(s-t) \psi=U \text {. }
$$

By integrating both sides of the last equality above, we conclude that

$$
u(z, F)=\alpha F(z)^{s-t},
$$

for some $\alpha \in \mathbb{C} \backslash\{0\}$. Therefore, by combining the representations of $F, B, A, g$ with (53), we conclude that

$$
\prod_{j=0}^{n} f\left(z+c_{j}\right)^{\lambda_{j}}=\alpha \frac{a_{s}(z)}{b_{t}(z)}(f \circ p)^{s-t} .
$$

If $s t \neq 0$, we deduce from (11) and (54) that

$$
\begin{aligned}
& \alpha \frac{a_{s}(z)}{b_{t}(z)}(f \circ p)^{s-t} \\
& \quad=R(z, f \circ p) \\
& \quad=\frac{a_{0}(z)+a_{1}(z)(f \circ p)+\cdots+a_{s}(z)(f \circ p)^{s}}{b_{0}(z)+b_{1}(z)(f \circ p)+\cdots+b_{t}(z)(f \circ p)^{t}} .
\end{aligned}
$$

From this, we get that $R(z, f \circ p)$ is not irreducible in $f \circ p$, a contradiction. Thus, $t=0$ or $s=0$. Therefore, we deduce from (54) that

$$
\prod_{j=0}^{n} f\left(z+c_{j}\right)^{\lambda_{j}}=\alpha \frac{a_{s}(z)}{b_{0}(z)}(f \circ p)^{s}
$$

or

$$
\prod_{j=0}^{n} f\left(z+c_{j}\right)^{\lambda_{j}}=\alpha \frac{a_{0}(z)}{b_{t}(z)} \frac{1}{(f \circ p)^{t}} .
$$

The proof of Theorem 3 is completed.

Proof of Theorem 5. Assume first that $f(z)=D(z) e^{E(z)}$, where $D(z)$ is a rational function and $E(z)$ is a polynomial. One can see from (17) that

$$
\begin{aligned}
c(z) & =f(z)^{m} \prod_{i=0}^{n} f\left(z+c_{i}\right)^{\lambda_{i}} \\
& =\left[D(z)^{m} \prod_{i=0}^{n} D\left(z+c_{i}\right)^{\lambda_{i}}\right] e^{m E(z)+\sum_{i=0}^{n} \lambda_{i} E\left(z+c_{i}\right)} \\
& :=G(z) e^{M(z)}
\end{aligned}
$$

where $G(z)=D(z)^{m} \prod_{i=0}^{n} D\left(z+c_{i}\right)^{\lambda_{i}}$ is rational and $M(z)=$ $m E(z)+\sum_{i=0}^{n} \lambda_{i} E\left(z+c_{i}\right)$ is a polynomial.

Suppose next that $c(z)=G(z) e^{M(z)}$, where $G(z)$ is a rational function and $M(z)$ is a polynomial. Since $f(z)$ has only finitely many poles, we conclude from (17) that

$$
\begin{aligned}
N\left(r, \frac{1}{f}\right) & \leq N\left(r, \frac{1}{f^{m}}\right)=N\left(r, \frac{\prod_{i=0}^{n} f\left(z+c_{i}\right)^{\lambda_{i}}}{c(z)}\right) \\
& =O(\log r) .
\end{aligned}
$$

Thus, $f(z)$ has only finitely many zeros and poles, and $f(z)=D(z) e^{E(z)}$, where $D(z)$ is rational and $E(z)$ is an entire function. In the following, we only prove $E(z)$ is a polynomial. Now, substituting $f(z)=D(z) e^{E(z)}$ and $c(z)=G(z) e^{M(z)}$ into (17), we get

$$
\begin{aligned}
& \prod_{i=0}^{n}\left\{D\left(z+c_{i}\right)^{\lambda_{i}} \exp \left(\lambda_{i} E\left(z+c_{i}\right)\right)\right\} \\
& =\frac{G(z)}{D(z)^{m}} \exp (M(z)-m E(z)), \\
& \left(\prod_{i=0}^{n} D\left(z+c_{i}\right)^{\lambda_{i}}\right) \exp \left(\sum_{i=0}^{n} \lambda_{i} E\left(z+c_{i}\right)\right) \\
& =\frac{G(z)}{D(z)^{m}} \exp (M(z)-m E(z)) .
\end{aligned}
$$

Thus, we deduce from Lemma 20 that two exponents in (61) cancel each other to a constant $\tau \in \mathbb{C}$ such that

$$
\sum_{i=0}^{n} \lambda_{i} E\left(z+c_{i}\right)=M(z)-m E(z)+\tau ;
$$


that is,

$$
\sum_{i=1}^{n} \lambda_{i} E\left(z+c_{i}\right)+\left(\lambda_{0}+m\right) E(z)=M(z)+\tau .
$$

Suppose that $E(z)$ is not a polynomial. If $E(z)$ is a transcendental entire function of finite order, we get from Lemma 21, Remark 22, and (63) that $\sigma(E) \geq 1$. Otherwise, $E(z)$ is a transcendental entire function of infinite order. These both show that $\sigma(f)=\infty$, contradicting the assumption that $f(z)$ is finite order. Thus, $E(z)$ is a polynomial. The proof of Theorem 5 is completed.

\section{Proof of Theorem 7}

Lemmas 23 and 25 reveal some properties of the maximal module of the polynomial in composite function $f \circ p$ with a meromorphic function $f(z)$ and a polynomial $p(z)$, which are useful for proving the existence of Borel exceptional value of finite order meromorphic solutions of functional difference equation of type (19).

Lemma 23. Let $g(z)$ be a nonconstant entire function of order $\sigma(g)=\sigma<\infty$. Suppose that $\alpha_{j}(z)(j=1,2, \ldots, m)$ are small meromorphic functions relative to $g(z)$. Then there exists a set $E \subset(1, \infty)$ of lower logarithmic density 1 such that

$$
\frac{M\left(r, \alpha_{j}\right)}{M(r, g)} \longrightarrow 0, \quad j=1,2, \ldots, m,
$$

hold simultaneously for all $r \in E$ as $r \rightarrow \infty$, where the lower logarithmic density of set $E$ is defined by

$$
\underline{\log \operatorname{dens}}(E)=\liminf _{r \rightarrow \infty} \frac{\int_{[1, r] \cap E}(d t / t)}{\log r} .
$$

Remark 24. The proof of Lemma 23 is similar to the proof of Lemma 2.4 and Remark 2.5 in [16]. Here, we omit it.

Lemma 25. Let $f(z)$ be a finite order transcendental meromorphic function satisfying (12), and $p(z)=d_{k} z^{k}+\cdots+d_{1} z+$ $d_{0}$ is a polynomial with constant coefficients $d_{k}(\neq 0), \ldots, d_{1}, d_{0}$ and of the degree $k \geq 1$. Suppose that

$$
\begin{aligned}
H(z)= & a_{n}(z)(f \circ p)^{n}+a_{n-1}(z)(f \circ p)^{n-1} \\
& +\cdots+a_{1}(z)(f \circ p)+a_{0}(z)
\end{aligned}
$$

is a polynomial in $f \circ p$, where $n(\geq 1)$ is a positive integer and $a_{n}(z)(\equiv 0), a_{n-1}(z), \ldots, a_{1}(z), a_{0}(z)$ are small meromorphic functions relative to $f(z)$. Then there exists a set $E_{1}$ of lower logarithmic density 1 such that

$$
\log ^{+} M(r, H) \geq(n-2 \varepsilon) T\left(\mu r^{k}, f\right)
$$

for all $r \in E_{1}$ as $r \rightarrow \infty$, where $0<\mu<\left|d_{k}\right|$. Hence, $H(z) \not \equiv$ 0 .

Proof of Lemma 25. Let $\tau$ be the multiplicity of pole of $f(z)$ at the origin, and let $q(z)$ be a canonical product formed with the nonzero poles of $f(z)$. Since $f(z)$ satisfies (12), then $h(z)=z^{\tau} q(z)$ is an entire function. Thus, $g(z)=h(z) f(z)$ is entire, and (37), (38), and (40) also hold.

Now, substituting $f(z)=g(z) / h(z)$ into (66), we conclude that

$$
\begin{aligned}
H(z)= & a_{n}(z) \cdot \frac{(g \circ p)^{n}}{(h \circ p)^{n}}+a_{n-1}(z) \cdot \frac{(g \circ p)^{n-1}}{(h \circ p)^{n-1}}+\cdots \\
& +a_{1}(z) \cdot \frac{(g \circ p)}{(h \circ p)}+a_{0}(z) \\
= & \frac{a_{n}(z)}{(h \circ p)^{n}}(g \circ p)^{n} \\
& \times\left[1+\frac{a_{n-1}(z)(h \circ p)}{a_{n}(z)}(g \circ p)^{-1}+\cdots\right. \\
& +\frac{a_{1}(z)(h \circ p)^{n-1}}{a_{n}(z)}(g \circ p)^{1-n} \\
& \left.+\frac{a_{0}(z)(h \circ p)^{n}}{a_{n}(z)}(g \circ p)^{-n}\right] .
\end{aligned}
$$

We note from Lemma 15 and (40) that

$$
\begin{gathered}
T\left(r, \frac{a_{n}(z)}{(h \circ p)^{n}}\right)=S(r, g \circ p), \\
T\left(r, \frac{a_{j}(z)(h \circ p)^{n-j}}{a_{n}(z)}\right)=S(r, g \circ p) \\
\text { for } j=0,1, \ldots, n-1 .
\end{gathered}
$$

Therefore, we deduce from Lemma 23 that there exists a set $E \subset(1, \infty)$ of lower logarithmic density 1 such that

$$
\begin{gathered}
\frac{M\left(r, a_{n}(z) /(h \circ p)^{n}\right)}{M(r, g \circ p)} \longrightarrow 0, \\
\frac{M\left(r, a_{j}(z)(h \circ p)^{n-j} / a_{n}(z)\right)}{M(r, g \circ p)} \longrightarrow 0, \\
(j=0,1, \ldots, n-1) .
\end{gathered}
$$

Moreover, according to the choosing of $E$ in the proof of Lemma 23, we know that $a_{j}(z)(h \circ p)^{n-j} / a_{n}(z)$ for 
$j=0,1, \ldots, n-1$ have no zeros and poles for all $|z|=r \in E$. Thus, we conclude from (68) and (70) that, for any $\varepsilon>0$,

$$
\begin{aligned}
& M(r, H) \geq M(r, g \circ p)^{n-\varepsilon} \\
& \times\left[1-\left|\frac{a_{n-1}(z)(h \circ p)}{a_{n}(z)}\right| M(r, g \circ p)^{-1}-\cdots\right. \\
&-\left|\frac{a_{1}(z)(h \circ p)^{n-1}}{a_{n}(z)}\right| M(r, g \circ p)^{1-n} \\
&\left.-\left|\frac{a_{0}(z)(h \circ p)^{n}}{a_{n}(z)}\right| M(r, g \circ p)^{-n}\right] \\
& \geq(1-\varepsilon) M(r, g \circ p)^{n-\varepsilon},
\end{aligned}
$$

and so

$$
\log ^{+} M(r, H) \geq\left(n-\frac{3}{2} \varepsilon\right) \log ^{+} M(r, g \circ p)
$$

for all $|z|=r \in E$ and $|g \circ p(z)|=M(r, g \circ p)$.

Therefore, we deduce from Lemma 15 and (38) that

$$
\log ^{+} M(r, H) \geq(n-2 \varepsilon) T\left(\mu r^{k}, f\right)
$$

for all $|z|=r \in E_{1}=E \cap\left(r_{0},+\infty\right)$, where $r_{0}>0$. It is obvious that $E_{1}$ has lower logarithmic density 1. The proof of Lemma 25 is completed.

Proof of Theorem 7. Suppose that $f(z)$ has two finite Borel exceptional values $a$ and $b(\neq 0, a)$. For the case where one of $a$ and $b$ is infinite, we can use a similar method to prove it. Set

$$
g(z)=\frac{f(z)-a}{f(z)-b} .
$$

Then $\sigma(g)=\sigma(f)$ and

$$
\lambda(g)=\lambda(f-a)<\sigma(g), \quad \lambda\left(\frac{1}{g}\right)=\lambda(f-b)<\sigma(g) .
$$

It follows from (74) that

$$
f(z)=\frac{a-b g(z)}{1-g(z)} .
$$

Now, substituting (76) into (19), we conclude that

$$
\begin{aligned}
g(z+c)= & \left(\sum_{i=0}^{s} a_{i}(z)(a-b g \circ p)^{i}(1-g \circ p)^{s+t-i}\right. \\
& \left.-a \sum_{j=0}^{t} b_{j}(z)(a-b g \circ p)^{j}(1-g \circ p)^{s+t-j}\right)
\end{aligned}
$$

$$
\begin{gathered}
\times\left(\sum_{i=0}^{s} a_{i}(z)(a-b g \circ p)^{i}(1-g \circ p)^{s+t-i}\right. \\
\left.-b \sum_{j=0}^{t} b_{j}(z)(a-b g \circ p)^{j}(1-g \circ p)^{s+t-j}\right)^{-1} \\
=\left((-g \circ p)^{s+t}\left(\sum_{i=0}^{s} a_{i}(z) b^{i}-a \sum_{j=0}^{t} b_{j}(z) b^{j}\right)\right. \\
\left.+\cdots+\left(\sum_{i=0}^{s} a_{i}(z) a^{i}-a \sum_{j=0}^{t} b_{j}(z) a^{j}\right)\right) \\
\times\left((-g \circ p)^{s+t}\left(\sum_{i=0}^{s} a_{i}(z) b^{i}-b \sum_{j=0}^{t} b_{j}(z) b^{j}\right)\right. \\
\left.+\cdots+\left(\sum_{i=0}^{s} a_{i}(z) a^{i}-b \sum_{j=0}^{t} b_{j}(z) a^{j}\right)\right)^{-1} .
\end{gathered}
$$

Since $a_{0}(z)+a_{1}(z)(f \circ p)+\cdots+a_{s}(z)(f \circ p)^{s}$ and $b_{0}(z)+$ $b_{1}(z)(f \circ p)+\cdots+b_{t}(z)(f \circ p)^{t}$ are irreducible in $f \circ p$, we conclude that at least one of the following three inequalities holds; that is,

$$
\begin{aligned}
& \left(\sum_{i=0}^{s} a_{i}(z) b^{i}-a \sum_{j=0}^{t} b_{j}(z) b^{j}\right) \cdot\left(\sum_{i=0}^{s} a_{i}(z) b^{i}-b \sum_{j=0}^{t} b_{j}(z) b^{j}\right) \\
& \quad \neq 0, \\
& \left(\sum_{i=0}^{s} a_{i}(z) b^{i}-a \sum_{j=0}^{t} b_{j}(z) b^{j}\right) \cdot\left(\sum_{i=0}^{s} a_{i}(z) a^{i}-b \sum_{j=0}^{t} b_{j}(z) a^{j}\right) \\
& \quad \neq 0, \\
& \left(\sum_{i=0}^{s} a_{i}(z) b^{i}-b \sum_{j=0}^{t} b_{j}(z) b^{j}\right) \cdot\left(\sum_{i=0}^{s} a_{i}(z) a^{i}-a \sum_{j=0}^{t} b_{j}(z) a^{j}\right) \\
& \quad \neq 0 .
\end{aligned}
$$

Thus, we deduce from Theorem 3 that

$$
g(z+c)=c(z)(g \circ p)^{l},
$$

where $c(z)$ is meromorphic function satisfying $T(r, c)=$ $S(r, g)$ and $l \in \mathbb{Z}$. Clearly, $l \neq 0$ and $g(z)$ is of regular growth from (75); see [15, Staz 13.4]. Therefore, $\sigma(c)<\sigma(g)$.

If $l \geq 1$, we conclude from (77) and (79) that

$$
\begin{gathered}
(-1)^{s+t} c(z)\left(\sum_{i=0}^{s} a_{i}(z) b^{i}-b \sum_{j=0}^{t} b_{j}(z) b^{j}\right)(g \circ p)^{s+t+l} \\
+\cdots+\left(-\sum_{i=0}^{s} a_{i}(z) a^{i}+a \sum_{j=0}^{t} b_{j}(z) a^{j}\right)=0 .
\end{gathered}
$$


Thus, we deduce from Lemma 25 that (80) is a contradiction. If $l \leq-1$, we use the same method as above to get another contradiction. Therefore, $f(z)$ has at most one Borel exceptional value. The proof of Theorem 7 is completed.

\section{Proof of Theorems 9 and 14}

We first recall two lemmas.

Lemma 26 (see [17, Lemma 2.1]). Let $f(z)$ be a nonconstant meromorphic function, $s>0, \alpha<1$, and $F \subset \mathbb{R}^{+}$the set of all $r$ such that

$$
T(r, f) \leq \alpha T(r+s, f)
$$

If the logarithmic measure of $F$ is infinite, that is, $\int_{F}(d t / t)=\infty$, then $f(z)$ is of infinite order of growth.

Lemma 27 (see [18, Corollary 2.6] and [19, Corollary 2.2]). Let $f(z)$ be a meromorphic function of finite order, and let $c \in$ $\mathbb{C}$. Then

$$
m\left(r, \frac{f(z+c)}{f(z)}\right)=S(r, f)
$$

for all $r$ outside of a possible exceptional set of finite logarithmic measure.

Proof of Theorem 9. For any $\varepsilon(0<\varepsilon<(d-(n+$ $\left.\left.1) \operatorname{deg}_{f}(H)\right) /\left(d+(n+1) \operatorname{deg}_{f}(H)\right)\right)$, we may apply ValironMohon'ko lemma, Lemma 17, (5), and (21) to conclude that

$$
\begin{aligned}
& d(1-\varepsilon) T(r, f) \\
& \leq d T(r, f)+S(r, f) \\
&= T\left(r, \frac{a_{0}(z)+a_{1}(z) f(z)+\cdots+a_{s}(z) f(z)^{s}}{b_{0}(z)+b_{1}(z) f(z)+\cdots+b_{t}(z) f(z)^{t}}\right) \\
&= T(r, H(z, f(z))) \leq(n+1) \operatorname{deg}_{f}(H) T(r+C, f) \\
&+S(r, f) \\
& \leq(n+1) \operatorname{deg}_{f}(H)(1+\varepsilon) T(r+C, f),
\end{aligned}
$$

for all $r$ outside of a possible exceptional set of finite logarithmic measure.

Denote

$$
\alpha=\frac{(n+1) \operatorname{deg}_{f}(H)(1+\varepsilon)}{d(1-\varepsilon)} .
$$

Then $\alpha<1$ since $0<\varepsilon<\left(d-(n+1) \operatorname{deg}_{f}(H)\right) /(d+(n+$ $\left.1) \operatorname{deg}_{f}(H)\right)$ and $d>(n+1) \operatorname{deg}_{f}(H)$. Thus,

$$
T(r, f) \leq \alpha T(r+C, f)
$$

holds for all $r$ in a set with infinite logarithmic measure. Therefore, we deduce from Lemma 26 and (85) that $\sigma(f)=$ $\infty$. The proof of Theorem 9 is completed.
Proof of Theorem 14. Assume, contrary to the assertion, that $f(z)$ is meromorphic of finite order. Taking into account the assumption that $H(z, f(z))$ is homogeneous, we deduce from Lemma 27 that

$$
m\left(r, \frac{H(z, f(z))}{f(z)^{\operatorname{deg}_{f}(H)}}\right)=S(r, f)
$$

for all $r$ outside of a possible exceptional set of finite logarithmic measure.

Denote $C=\max _{1 \leq i \leq n}\left\{\left|c_{i}\right|\right\}$. Since $H(z)$ is homogeneous and has at least one difference monomial of type $\prod_{j=0}^{n} f(z+$ $\left.c_{j}\right)^{\lambda_{j}}$, we immediately conclude that, by looking at pole multiplicities, summing over $|z| \leq r$, and integrating logarithmically,

$$
\begin{aligned}
& N\left(r, \frac{H(z, f(z))}{f(z)^{\operatorname{deg}_{f}(H)}}\right) \\
& \quad \leq \kappa_{f}(H)\left(N(r+C, f)+N\left(r, \frac{1}{f}\right)\right)+S(r, f) \\
& \quad \leq \operatorname{deg}_{f}(H)\left(N(r+C, f)+N\left(r, \frac{1}{f}\right)\right)+S(r, f) .
\end{aligned}
$$

Therefore,

$$
\begin{aligned}
& T(r, H(z, f(z))) \\
&=m(r, H(z, f(z)))+N(r, H(z, f(z))) \\
& \leq m\left(r, \frac{H(z, f(z))}{f(z)^{\operatorname{deg}_{f}(H)}}\right)+m\left(r, f(z)^{\operatorname{deg}_{f}(H)}\right) \\
&+N\left(r, \frac{H(z, f(z))}{f(z)^{\operatorname{deg}_{f}(H)}}\right)+N\left(r, f(z)^{\operatorname{deg}_{f}(H)}\right) \\
& \leq \operatorname{deg}_{f}(H)\left(N(r+C, f)+N\left(r, \frac{1}{f}\right)\right) \\
&+T\left(r, f(z)^{\operatorname{deg}_{f}(H)}\right)+S(r, f) \\
& \leq 3 \operatorname{deg}_{f}(H) T(r+C, f)+S(r, f)
\end{aligned}
$$

for all $r$ outside of a possible exceptional set of finite logarithmic measure. The remainder can be proven by a similar method in Theorem 9. The proof of Theorem 14 is completed.

\section{Conflict of Interests}

The authors declare that there is no conflict of interests regarding the publication of this paper.

\section{Acknowledgments}

The authors are grateful to the referees for their helpful suggestions to improve this paper. The first author also thanks Professor Ilpo laine and Professor Risto Korhonen for 
their valuable suggestion to the present paper. Research is supported by National Natural Science Foundation of China (nos. 11171119 and 11171121) and Guangdong National Natural Science Foundation (no. S2012040006865).

\section{References}

[1] W. K. Hayman, Meromorphic Functions, Clarendon Press, Oxford, UK, 1964.

[2] M. J. Ablowitz, R. Halburd, and B. Herbst, "On the extension of the Painlevé property to difference equations," Nonlinearity, vol. 13, no. 3, pp. 889-905, 2000.

[3] B. Grammaticos, T. Tamizhmani, A. Ramani, and K. M. Tamizhmani, "Growth and integrability in discrete systems," Journal of Physics A, vol. 34, no. 18, pp. 3811-3821, 2001.

[4] J. Heittokangas, R. Korhonen, I. Laine, J. Rieppo, and K. Tohge, "Complex difference equations of Malmquist type," Computational Methods and Function Theory, vol. 1, no. 1, pp. 27-39, 2001.

[5] I. Laine, J. Rieppo, and H. Silvennoinen, "Remarks on complex difference equations," Computational Methods and Function Theory, vol. 5, no. 1, pp. 77-88, 2005.

[6] G. G. Gundersen, J. Heittokangas, I. Laine, J. Rieppo, and D. Yang, "Meromorphic solutions of generalized Schröder equations," Aequationes Mathematicae, vol. 63, no. 1-2, pp. 110135, 2002.

[7] W. G. Kelley and A. C. Peterson, Difference Equations, Academic Press, Boston, Mass, USA, 1991.

[8] I. Laine and C. C. Yang, "Clunie theorems for difference and q-difference polynomials," Journal of the London Mathematical Society, vol. 76, no. 3, pp. 556-566, 2007.

[9] R. Goldstein, "Some results on factorisation of meromorphic functions," Journal of the London Mathematical Society, vol. 4, pp. 357-364, 1971.

[10] V. I. Gromak, I. Laine, and S. Shimomura, Painlevé Differential Equations in the Complex Plane, vol. 28, Walter de Gruyter, Berlin, Germany, 2002.

[11] G. G. Gundersen, "Finite order solutions of second order linear differential equations," Transactions of the American Mathematical Society, vol. 305, no. 1, pp. 415-429, 1988.

[12] R. Goldstein, "On meromorphic solutions of certain functional equations," Aequationes Mathematicae, vol. 18, no. 1-2, pp. 112$157,1978$.

[13] C. C. Yang and H. X. Yi, Uniqueness Theory of Meromorphic Functions, vol. 557, Kluwer Academic Publishers Group, Dordrecht, The Netherlands, 2003.

[14] Z. X. Chen and K. H. Shon, "On growth of meromorphic solutions for linear difference equations," Abstract and Applied Analysis, vol. 2013, Article ID 619296, 6 pages, 2013.

[15] G. Jank and L. Volkmann, Einführung in die Theorie der ganzen und meromorphen Funktionen mit Anwendungen auf Differentialgleichungen, Birkhäuser, Basel, Switzerland, 1985.

[16] J. Wang, "Growth and poles of meromorphic solutions of some difference equations," Journal of Mathematical Analysis and Applications, vol. 379, no. 1, pp. 367-377, 2011.

[17] R. G. Halburd and R. J. Korhonen, "Finite-order meromorphic solutions and the discrete Painlevé equations," Proceedings of the London Mathematical Society, vol. 94, no. 2, pp. 443-474, 2007.

[18] Y. M. Chiang and S. J. Feng, "On the Nevanlinna characteristic of $f(z+\eta)$ and difference equations in the complex plane," Ramanujan Journal, vol. 16, no. 1, pp. 105-129, 2008.
[19] R. G. Halburd and R. J. Korhonen, "Difference analogue of the lemma on the logarithmic derivative with applications to difference equations," Journal of Mathematical Analysis and Applications, vol. 314, no. 2, pp. 477-487, 2006. 


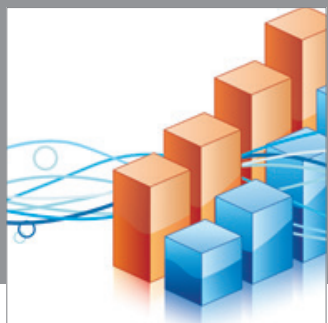

Advances in

Operations Research

mansans

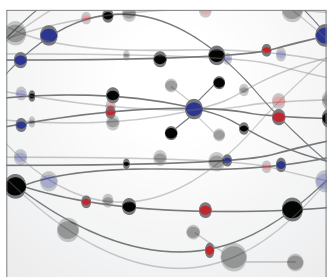

The Scientific World Journal
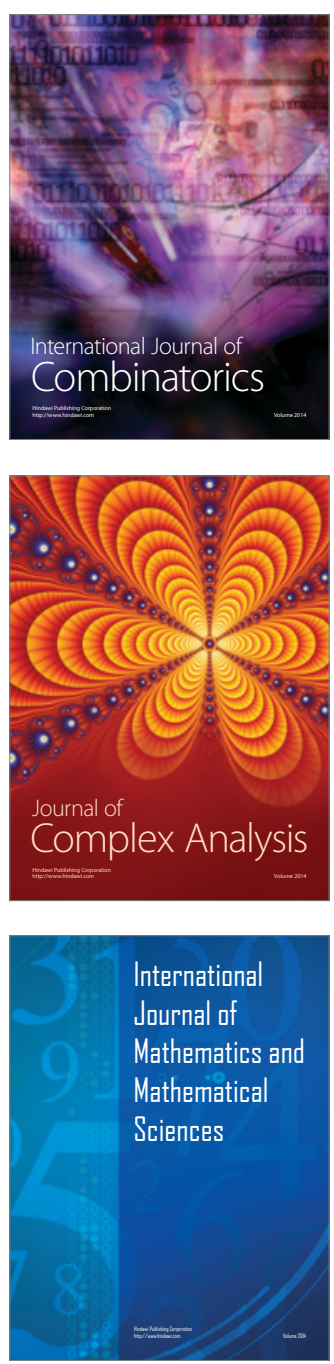
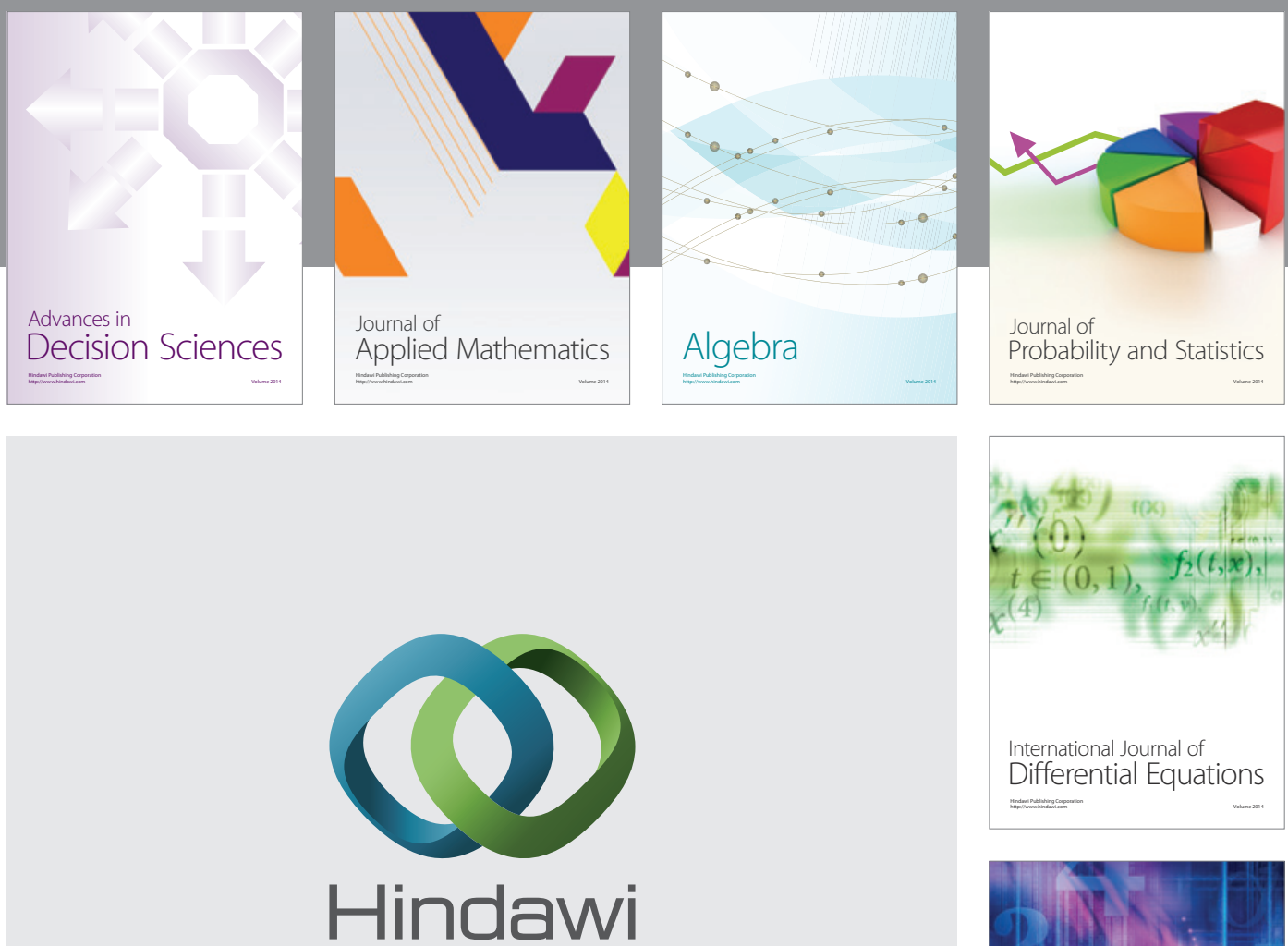

Submit your manuscripts at http://www.hindawi.com
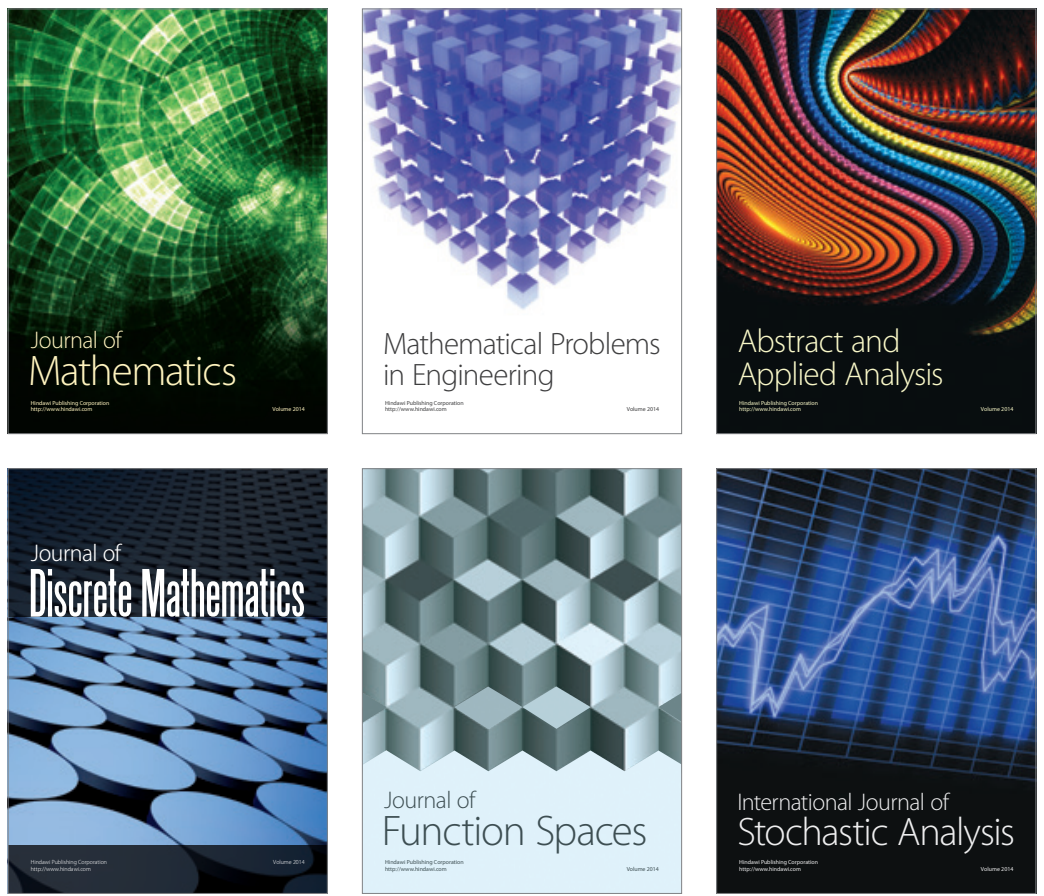

Journal of

Function Spaces

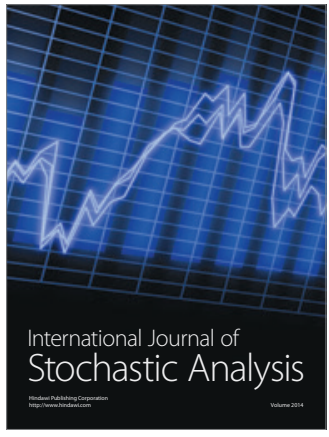

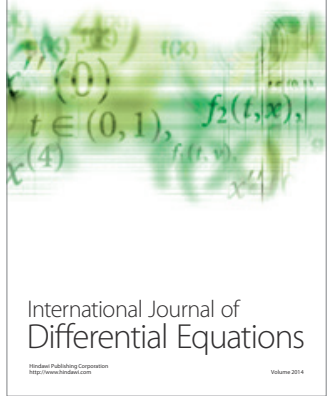
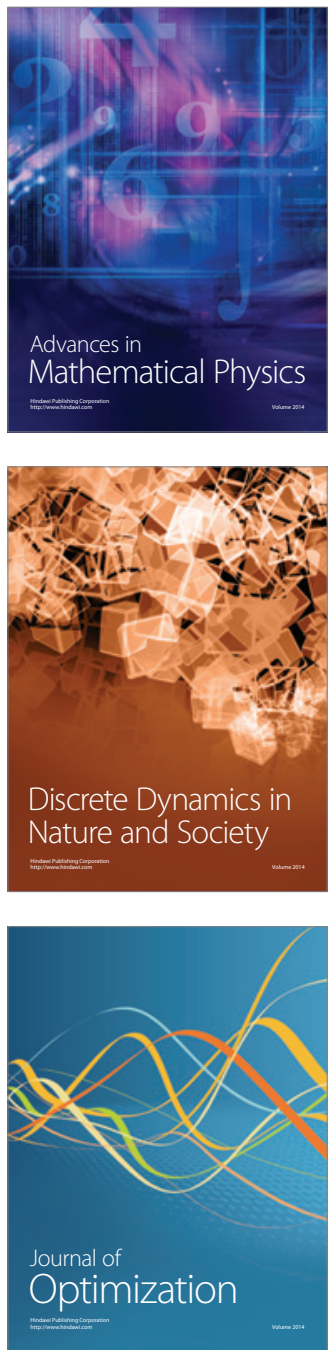